\title{
Ulinastatin treatment and renal injury in patients undergoing aortic valve replacement with cardiopulmonary bypass. A note of aution
}

\section{To the Editor}

I have read with great interest the manuscript by Oh et al. [1], presenting the use of ulinastatin as a possible method to protect the kidney during surgery with cardiopulmonary baypass (CPB).

The authors use ulinastatin, a protease inhibitor which may supress inflammatory reactions as well as the noxious effects that free radical elimination may produce in the cells of the kidney.

I would like to add some comments to the discussion:

The damage imposed to the kidneys during cardiac surgery is multifactorial in its origin. Of course CPB has been blamed as the main factor, but there are others whose relevance is as important as the CPB itself: changes in hematocrit and temperature, use of vasoactive and nephrotoxic drugs, hemodynamic changes, low cardiac output, just to mention a few. It seems interesting then some groups have reported that the incidence of renal damage during coronary surgery off pump is at least similar to those cases performed with CPB [2].

So, specific damage to the kidneys by $\mathrm{CPB}$ remains to be elucidated.

Renal dysfunction following cardiac surgery with CPB has a relatively low incidence in patients with normal preoperative dysfunction. This is the case for this study. The numbers of patients in each group is too small to obtain a difference, if it exists.

There are two groups of patients at the highest risk for developing renal dysfunction after cardiac surgery: those whohave preoperative abnormal renal function (plasma creatinine level 1.5 or higher) or those with hemodynamic instability; recent myocardial infarction, use of preoperative vasoactive drugs (those with alpha effects), low cardiac output, use of intra-aortic balloon pump, sepsis among others. These are the groups that should be chosen to be studied. Unfortunately those groups are quite difficult to study due to the inherent conditions of their clinical state.

In my point of view, the study with patients with normal preoperative renal function is a dead end.

Different groups have worked for many years trying to find a therapy that may protect the kidney against insults during cardiac and non-cardiac surgery: dopamine, furosemide, fenoldopan, atrial natriuretic factor, sodium bicarbonate, n-acetyl cysteine, enoximone, dexmedetomidine, high or low hematocrit, high or low temperatures, among others. To my knowledge that therapy has not been found as of today [3].

When we talk about "renal protection", is this phrase the same as "brain protection" or "heart protection"? It is my fear that we are trying to find techniques that may keep the kidney working well during the insult, rather than looking for something that may reduce oxygen compsumption during the damage.

Just a thought.

In the meantime, results of this study should be analyzed very carefully.

\section{Guillermo Lema}

Division of Anesthesiology, Pontificia Universidad Católica de Chile, Marcoleta 367, Santiago, Chile

E-mail: glema@med.puc.cl

(C) This is an open-access article distributed under the terms of the Creative Commons Attribution Non-Commercial License (http:// creativecommons.org/licenses/by-nc/3.0/), which permits unrestricted non-commercial use, distribution, and reproduction in any medium, provided the original work is properly cited. 


\section{References}

1. Oh SY, Kim JC, Choi YS, Lee WK, Lee YK, Kwak YL. Effects of ulinastatin treatment onmyocardial and renal injury in patients undergoing aortic valve replacement with cardiopulmonary bypass. Korean J Anesthesiol 2012; 62: 148-53.

2. Elmistekawy E, Chan V, Bourke ME, Dupui JY, Rubens FD, Mesana TG, et al. Off-pump coronary artery bypass grafting does not preserve renal function better than on-pump coronary artery bypass grafting: results of a case-matched study. J Thorac Cardiovasc Surg 2012; 143: 85-92.

3. Bove T, Landoni G, Calabro MG, Aletti G, Marino G, Cerchierini E, et al. Renoprotective action of fenoldopam in high-risk patients undergoing cardiac surgery. Circulation 2005; 111: 3230-5.

\section{In Response}

We appreciate the constructive comments by Dr. Guillermo Lema regarding our article. Undoubtedly, patients with preexisting renal dysfunction would be more prone to aggravation of the renal dysfunction after cardiac surgery. Theoretically, these patients would benefit the most from therapeutic measures aimed at reducing the postoperative kidney injury, yet it would also require a harder clinical endpoint such as the requirement of renal replacement therapy for validation. As the incidence of renal replacement therapy after cardiac surgery is low, this would require a substantial number of patients.

In our study, we used differences in renal biomarkers as the primary endpoint in a relatively homogenous group of patients undergoing aortic valve replacement. Compared to other valvular procedures, aortic valve surgery is associated with relatively higher incidence of acute kidney injury [1]. As with the assessment of postoperative myocardial infarction using cardiac enzymes, the correlation between renal dysfunction and changes in renal biomarkers, especially creatinine, has been well validated [2]. Even transient elevations in the postoperative serum creatinine concentrations have been shown to be associated with adverse outcome following cardiac surgery. As this was the first study to address the reno-protective effect of ulinastatin, we tried to avoid the introduction of confounding factors as much as possible. In patients with pre-existing renal dysfunction, however, the concentrations of renal biomarkers would be already increased and diverse, which could act as a major confounder. Thus, to efficiently assess the potential reno-protective effect of ulinastatin using a small sample size, we had excluded patients with pre-existing renal dysfunction. This in turn, however, invariably confronts generalization of the observed results as was commented by Dr. Guillermo Lema.

Notwithstanding the complex relationship between preoperative patient's co-morbid diseases and postoperative renal dysfunction and clinical outcome, we do acknowledge that a further study with a large sample size involving a heterogeneous group of patients would be necessary to draw a more definite conclusion adding value to the literature. We hope that our study would draw many attentions in that regard.

Se-Young $\mathrm{Oh}^{1}$, Jong Chan $\mathrm{Kim}^{2}$, Yong Seon $\mathrm{Choi}^{1}$, Woo Kyung Lee $^{1}$, Yeong-Kyu Lee ${ }^{1}$, Young Lan Kwak ${ }^{1}$

Department of Anesthesiology and Pain Medicine, ${ }^{\mathrm{l} Y o n s e i ~ U n i v e r s i t y ~}$ College of Medicine, Seoul, ${ }^{2}$ Kyungpook National University Hospital, Daegu, Korea

\section{Yong Seon Choi}

Department of Anesthesiology and Pain Medicine, Yonsei University College of Medicine, Seoul, Korea

E-mail: yschoi@yuhs.ac

\section{References}

1. Stafford-Smith M, Patel UD, Phillips-Bute BG, Shaw AD, Swaminathan M. Acute kidney injury and chronic kidney disease after cardiac surgery. Adv Chronic Kidney Dis 2008; 15: 257-77.

2. Ho J, Reslerova M, Gali B, Nickerson PW, Rush DN, Sood MM, et al. Serum creatinine measurement immediately after cardiac surgery and prediction of acute kidney injury. Am J Kidney Dis 2012; 59: 196-201. 